\title{
SOME EXTENSION OF THE BESSEL-TYPE ORTHOGONAL POLYNOMIALS.
}

\author{
J. Arvesú, R. Álvarez-Nodarse, F. Marcellán, and K. H. Kwon \\ Preprint MA/UC3M/8/1997
}

\begin{abstract}
Key words and phrases: Orthogonal polynomials, Bessel polynomials,
hypergeometric function, Perturbed orthogonal polynomials
\end{abstract}

AMS (MOS) subject classification: 33C45, 33A65, $42 \mathrm{C05}$.

\begin{abstract}
We consider the perturbation of the classical Bessel moment functional by the addition of the linear functional $M_{0} \delta(x)+M_{1} \delta^{\prime}(x)$, where $M_{0}$ and $M_{1} \in \mathbb{R}$. We give necessary and sufficient conditions in order for this functional to be a quasi-definite functional. In such a situation we analyze the corresponding sequence of monic orthogonal polynomials $B_{n}^{\alpha, M_{0}, M_{1}}(x)$. In particular, a hypergeometric representation $\left({ }_{4} F_{2}\right)$ for them is obtained.

Furthermore, we deduce a relation between the corresponding Jacobi matrices, as well as the asymptotic behavior of the ratio $B_{n}^{\alpha, M_{0}, M_{1}}(x) / B_{n}^{\alpha}(x)$, outside of the closed contour $\Gamma$ containing the origin and the difference between the new polynomials and the classical ones, inside $\Gamma$.
\end{abstract}

\section{Introduction.}

In this paper we will study a generalization of the Bessel polynomials [8], [6], which results when we perturb the classical Bessel moment functional by the addition of the linear functional $M_{0} \delta(x)+M_{1} \delta^{\prime}(x)$, where $M_{0}$ and $M_{1} \in \mathbb{R}$. If $M_{1}=0$, the corresponding sequence of orthogonal polynomials has been studied by E. Hendriksen [7] in the framework of rational approximation and by F. Marcellán and P. Maroni [10] in the theory of semi-classical orthogonal polynomials. There the corresponding second order differential equation is deduced.

When $M_{0}=0$ and $M_{1}>0$, a general theory is introduced in [5], but unfortunately only one example based in Hermite polynomials is considered.

Later on, in [1]-[2] the perturbation of the Laguerre linear functional by the addition of a linear functional $M_{0} \delta(x)+M_{1} \delta^{\prime}(x)$ is analyzed. There, the authors study asymptotic properties of the related orthogonal polynomials, the distribution of their zeros, the hypergeometric representation, and the second order differential equation that such polynomials satisfy.

In [3] the bounded case is studied, i.e., the perturbation of the Jacobi moment functional in the form,

$$
<\mathcal{U}, P>=\int_{-1}^{1} P(x)(1-x)^{\alpha}(1+x)^{\beta} d x+A_{0} P(1)+B_{0} P(-1)+A_{1} P^{\prime}(1)+B_{1} P^{\prime}(-1),
$$

where $P \in \mathbb{P}$ (linear space of polynomials with real coefficients), $\alpha, \beta>-1$ and $A_{0}, B_{0}, A_{1}, B_{1} \in \mathbb{R}$. We give necessary and sufficient conditions in order for $\mathcal{U}$ to be a quasi-definite moment functional. In such a situation we analyze the corresponding sequence of monic orthogonal polynomials $P_{n}^{\alpha, \beta, A_{0}, B_{0}, A_{1}, B_{1}}(x)$. In particular, a hypergeometric representation $\left({ }_{6} F_{5}\right)$, the three-term recurrence relation, the second 
order differential equation and a symmetry property are also obtained.

The interest of the Bessel-type orthogonal polynomials is twofold.

First, they are related with a quasi-definite moment functional. As a consequence, positivity does not play the main role as in the standard cases. Thus, the existence of a sequence of orthogonal polynomials is not guaranteed.

Second, it is well known that zeros of orthogonal polynomials are eigenvalues of a Jacobi symmetric matrix. Now, the real symmetry is lost and the properties of zeros change very much in the sense that a new distribution appears.

The structure of the paper is as follows. In Section 2 we list some of the main properties of the classical Bessel polynomials which will be used later on. In Section 3 we define the generalized polynomials and find some of their properties. In Section 4 we obtain the representation of the generalized Bessel polynomials in terms of the hypergeometric functions. In Section 5 we obtain an asymptotic formula for these polynomials and in Section 6 we establish their quasi-orthogonality. Finally, in Sections 7 and 8 we obtain the three-term recurrence relation that such polynomials satisfy as well as the corresponding Jacobi matrices.

\section{Bessel polynomials.}

In this section we have enclosed some formulas for the Bessel polynomials $\left\{B_{n}^{\alpha}(x)\right\}_{0}^{\infty}$ which will be useful to obtain the generalized polynomials orthogonal with respect to the quasi-definite moment functional (see Eq. (21) below). All the formulas and properties for the classical Bessel polynomials can be found in [6] and [12]. In this work we will use monic polynomials, i.e., polynomials with leading coefficients equal to 1 .

The Bessel polynomials are the polynomial solution of the second order linear differential equation (SODE) of hypergeometric type

$$
\sigma(x) y^{\prime \prime}(x)+\tau(x) y^{\prime}(x)+\lambda_{n} y(x)=0,
$$

where

$$
\sigma(x)=x^{2}, \quad \tau(x)=[(\alpha+2) x+2], \quad \lambda_{n}=-n(n+\alpha+1) .
$$

They are orthogonal with respect to the quasi-definite moment functional $\mathcal{L}_{0}$, associated with the weight function $\rho_{0}^{\alpha}$, on the linear space $\mathbb{P}$ of polynomials with real coefficients defined by

$$
<\mathcal{L}_{0}, P(x)>=\int_{\Gamma} P(z) \rho_{0}^{\alpha}(z) d z, \quad P \in \mathbb{P} .
$$

Here the integration is around the unit circle $\Gamma$ or around some closed contour in $\mathbb{C}$ containing the origin, and

$$
\rho_{0}^{\alpha}(z)=2^{\alpha+1} \sum_{m=0}^{\infty} \frac{(-2)^{m}}{\Gamma(m+\alpha+1) z^{m}}, \quad \alpha \neq-1,-2,-3, \ldots .
$$

The orthogonality relation is

$$
\frac{1}{2 \pi i} \int_{\Gamma} B_{n}^{\alpha}(z) B_{m}^{\alpha}(z) \rho_{0}^{\alpha}(z) d z=d_{n}^{2} \delta_{n m},
$$

where

$$
d_{n}^{2}=\left\|B_{n}^{\alpha}(x)\right\|^{2}=\frac{(-1)^{n+1} 2^{2 n+\alpha+1} \Gamma(n+\alpha+1) n !}{(2 n+\alpha+1) \Gamma(2 n+\alpha+2)} .
$$


They satisfy the differential relation

$$
\frac{d}{d x} B_{n}^{\alpha}(x)=n B_{n-1}^{\alpha+2}(x)
$$

Now, from the structure relation

$$
x^{2} \frac{d}{d x} B_{n}^{\alpha}(x)=n\left[x-\frac{2}{2 n+\alpha}\right] B_{n}^{\alpha}(x)+\frac{4 n(n+\alpha)}{(2 n+\alpha)^{2}(2 n+\alpha-1)} B_{n-1}^{\alpha}(x),
$$

and the three-term recurrence relation,

$$
x B_{n}^{\alpha}(x)=B_{n+1}^{\alpha}(x)+\beta_{n}^{\alpha} B_{n}^{\alpha}(x)+\gamma_{n}^{\alpha} B_{n-1}^{\alpha}(x), \quad n \geq 1,
$$

with

$$
\beta_{n}^{\alpha}=-\frac{2 \alpha}{(2 n+\alpha)(2 n+\alpha+2)}, \quad \gamma_{n}^{\alpha}=-\frac{4 n(n+\alpha)}{(2 n+\alpha+1)(2 n+\alpha)^{2}(2 n+\alpha-1)},
$$

and

$$
B_{0}^{\alpha}(x)=1, \quad B_{1}^{\alpha}(x)=x+\frac{2}{\alpha+2}
$$

we deduce

$$
B_{n-1}^{\alpha}(x)=\frac{(2 n+\alpha)^{2}(2 n+\alpha-1)}{4 n(n+\alpha)} x^{2}\left(B_{n}^{\alpha}\right)^{\prime}(x)+\frac{(2 n+\alpha)^{2}(2 n+\alpha-1)}{4(n+\alpha)}\left[\frac{2}{2 n+\alpha}-x\right] B_{n}^{\alpha}(x),
$$

which allows us to express the Bessel kernels in a polynomial form. If we take derivatives in the above formula we obtain,

$\left(B_{n-1}^{\alpha}\right)^{\prime}(x)=-\frac{(2 n+\alpha)^{2}(2 n+\alpha-1)}{4(n+\alpha)}\left[B_{n}^{\alpha}(x)+\left(x-\frac{2 x}{n}-\frac{2}{(2 n+\alpha)}\right)\left(B_{n}^{\alpha}\right)^{\prime}(x)-\frac{x^{2}}{n}\left(B_{n}^{\alpha}\right)^{\prime \prime}(x)\right]$.

The Christoffel-Darboux formula for Bessel polynomials $B_{n}^{\alpha}(x)$ is

$$
K_{n-1}^{\alpha}(x, y):=\sum_{k=0}^{n-1} \frac{B_{k}^{\alpha}(x) B_{k}^{\alpha}(y)}{d_{k}^{2}}=\frac{1}{x-y} \frac{B_{n}^{\alpha}(x) B_{n-1}^{\alpha}(y)-B_{n-1}^{\alpha}(x) B_{n}^{\alpha}(y)}{d_{n-1}^{2}}, \quad n=1,2,3, \ldots .
$$

Through the work we will denote by

$$
K_{n}^{\alpha(p, q)}(x, y)=\frac{\partial^{p+q}}{\partial x^{p} \partial y^{q}} K_{n}^{\alpha}(x, y)=\sum_{m=0}^{n} \frac{\left(B_{m}^{\alpha}\right)^{(p)}(x)\left(B_{m}^{\alpha}\right)^{(q)}(y)}{d_{m}^{2}},
$$

the kernels of the Bessel polynomials, as well as their derivatives with respect to $x$ and $y$, respectively.

The explicit representation of these polynomials is

$$
B_{n}^{\alpha}(x)=\frac{2^{n}}{(\alpha+n+1)_{n}} \sum_{k=0}^{n}\left(\begin{array}{c}
n \\
k
\end{array}\right)(n+\alpha+1)_{k}\left(\frac{x}{2}\right)^{k}, \quad B_{n}^{\alpha}(0)=\frac{2^{n}}{(\alpha+n+1)_{n}}>0
$$

or, equivalently, in terms of hypergeometric series

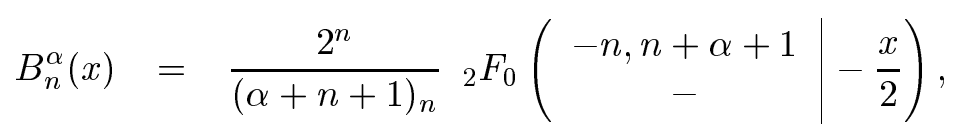

with

$$
{ }_{p} F_{q}\left(\begin{array}{c}
a_{1}, a_{2}, \ldots, a_{p} \\
b_{1}, b_{2}, \ldots, b_{q}
\end{array} \mid x\right)=\sum_{k=0}^{\infty} \frac{\left(a_{1}\right)_{k}\left(a_{2}\right)_{k} \cdots\left(a_{p}\right)_{k}}{\left(b_{1}\right)_{k}\left(b_{2}\right)_{k} \cdots\left(b_{q}\right)_{k}} \frac{x^{k}}{k !}
$$


Here $(\alpha)_{k}$ denotes the shifted factorial or Pochhammer's symbol:

$$
(\alpha)_{0}:=1, \quad(\alpha)_{k}:=\alpha(\alpha+1)(\alpha+2) \cdots(\alpha+k-1)=\frac{\Gamma(\alpha+k)}{\Gamma(\alpha)}, \quad k=1,2,3, \ldots .
$$

From the above properties, we deduce the following formulas which will be useful later on:

$$
\begin{aligned}
K_{n-1}^{\alpha}(x, 0) & =\frac{(2 n+\alpha)^{2}(2 n+\alpha-1) B_{n}^{\alpha}(0)}{4 n(n+\alpha) d_{n-1}^{2}}\left[n B_{n}^{\alpha}(x)-x\left(B_{n}^{\alpha}(x)\right)^{\prime}\right], \\
K_{n-1}^{\alpha(0,1)}(x, 0) & =\frac{(2 n+\alpha)^{2}(2 n+\alpha-1) B_{n}^{\alpha}(0)}{8 n(n+\alpha) d_{n-1}^{2}} \times \\
& \times\left[n^{2}(n+\alpha+1) B_{n}^{\alpha}(x)-(2+n(n+\alpha+1) x)\left(B_{n}^{\alpha}(x)\right)^{\prime}\right] \\
K_{n-1}^{\alpha(1,1)}(x, 0) & =\frac{(2 n+\alpha)^{2}(2 n+\alpha-1) B_{n}^{\alpha}(0)}{8 n(n+\alpha) d_{n-1}^{2}} \times \\
& \times\left[n(n-1)(n+\alpha+1)\left(B_{n}^{\alpha}(x)\right)^{\prime}-(2+n(n+\alpha+1) x)\left(B_{n}^{\alpha}(x)\right)^{\prime \prime}\right]
\end{aligned}
$$

and

$$
\begin{aligned}
K_{n-1}^{\alpha}(0,0) & =\frac{(2 n+\alpha)^{2}(2 n+\alpha-1)\left(B_{n}^{\alpha}(0)\right)^{2}}{4(n+\alpha) d_{n-1}^{2}} \\
K_{n-1}^{\alpha(0,1)}(0,0) & =\frac{(2 n+\alpha)^{2}(2 n+\alpha-1)(n-1) B_{n}^{\alpha}(0)\left(B_{n}^{\alpha}\right)^{\prime}(0)}{4 n(n+\alpha) d_{n-1}^{2}} \\
K_{n-1}^{\alpha(1,1)}(0,0) & =\frac{(2 n+\alpha)^{2}(2 n+\alpha-1)[n(n-2)(n+\alpha+1)+\alpha+2] B_{n}^{\alpha}(0)\left(B_{n}^{\alpha}\right)^{\prime}(0)}{8 n(n+\alpha) d_{n-1}^{2}}
\end{aligned}
$$

where

$$
B_{n}^{\alpha}(0)=\frac{2^{n}}{(n+\alpha+1)_{n}}, \quad\left(B_{n}^{\alpha}\right)^{\prime}(0)=\frac{n 2^{n-1}}{(n+\alpha+2)_{n-1}}
$$

\section{The definition and orthogonal relation.}

Let $\mathcal{L}_{0}$ be the quasi-definite moment functional (4) and $\left\{B_{n}^{\alpha}(x)\right\}_{0}^{\infty}$ the monic Bessel orthogonal polynomial sequence (MOPS) with respect to $\mathcal{L}_{0}$. Consider the perturbation $\mathcal{L}_{1}$ of $\mathcal{L}_{0}$ given by

$$
<\mathcal{L}_{1}, P(x)>=\int_{\Gamma} P(z) \rho_{0}^{\alpha}(z) d z+M_{0} P(0)-M_{1} P^{\prime}(0), \quad P \in \mathbb{P} .
$$

Proposition 1 The perturbed moment functional $\mathcal{L}_{1}$ is quasi-definite if and only if the determinant of the matrix $\mathbb{K}_{n}$, denoted by $\triangle_{n}^{\alpha, M_{0}, M_{1}}$, does not vanish for every $n \geq 0$, where

$$
\mathbb{K}_{n}=\left(\begin{array}{cc}
1+M_{0} K_{n}^{\alpha}(0,0)-M_{1} K_{n}^{\alpha(0,1)}(0,0) & -M_{1} K_{n}^{\alpha}(0,0) \\
M_{0} K_{n}^{\alpha(0,1)}(0,0)-M_{1} K_{n}^{\alpha(1,1)}(0,0) & 1-M_{1} K_{n}^{\alpha(0,1)}(0,0)
\end{array}\right), \quad n \geq 0 .
$$

Proof: Assume $\mathcal{L}_{1}$ is quasi-definite, and let $\left\{B_{n}^{\alpha, M_{0}, M_{1}}(x)\right\}_{0}^{\infty}$ be the MOPS with respect to $\mathcal{L}_{1}$. Then, we will prove that

$$
<\mathcal{L}_{1},\left(B_{n}^{\alpha, M_{0}, M_{1}}(x)\right)^{2}>=\frac{\triangle_{n}^{\alpha, M_{0}, M_{1}}}{\triangle_{n-1}^{\alpha, M_{0}, M_{1}}}<\mathcal{L}_{0},\left(B_{n}^{\alpha}(x)\right)^{2}>, \quad n \geq 0, \quad\left(\triangle_{-1}^{\alpha, M_{0}, M_{1}}=1\right)
$$


as well as

$$
\begin{aligned}
B_{n}^{\alpha, M_{0}, M_{1}}(x) & =B_{n}^{\alpha}(x)-M_{0} B_{n}^{\alpha, M_{0}, M_{1}}(0) K_{n-1}^{\alpha}(x, 0)+M_{1}\left(B_{n}^{\alpha, M_{0}, M_{1}}\right)^{\prime}(0) K_{n-1}^{\alpha}(x, 0)+ \\
& +M_{1} B_{n}^{\alpha, M_{0}, M_{1}}(0) K_{n-1}^{\alpha(0,1)}(x, 0),
\end{aligned}
$$

where $B_{n}^{\alpha, M_{0}, M_{1}}(0)$ and $\left(B_{n}^{\alpha, M_{0}, M_{1}}\right)^{\prime}(0)$ are given by

$$
\left(\begin{array}{c}
B_{n}^{\alpha, M_{0}, M_{1}}(0) \\
\left(B_{n}^{\alpha, M_{0}, M_{1}}\right)^{\prime}(0)
\end{array}\right)=\mathbb{K}_{n-1}^{-1}\left(\begin{array}{c}
B_{n}^{\alpha}(0) \\
\left(B_{n}^{\alpha}\right)^{\prime}(0)
\end{array}\right), \quad n \geq 1
$$

i.e.,

$$
\begin{gathered}
B_{n}^{\alpha, M_{0}, M_{1}}(0)=\frac{2 B_{n}^{\alpha}(0)\left[2+M_{1}(n+\alpha+1) K_{n-1}^{\alpha}(0,0)\right]}{4+n(n+\alpha+1)\left[M_{1} K_{n-1}^{\alpha}(0,0)\right]^{2}+4\left[M_{0}+M_{1}(n-1)(n+\alpha+1)\right] K_{n-1}^{\alpha}(0,0)}, \\
\left(B_{n}^{\alpha, M_{0}, M_{1}}\right)^{\prime}(0)=\frac{(n+\alpha+1) B_{n}^{\alpha}(0)\left[2 n+\left(2 M_{0}-M_{1}\left[n^{2}+n \alpha+n-\alpha-1\right]\right) K_{n-1}^{\alpha}(0,0)\right]}{4+n(n+\alpha+1)\left[M_{1} K_{n-1}^{\alpha}(0,0)\right]^{2}+4\left[M_{0}+M_{1}(n-1)(n+\alpha+1)\right] K_{n-1}^{\alpha}(0,0)} .
\end{gathered}
$$

In fact, we can write the Fourier expansion of the generalized Bessel polynomials in terms of the Bessel polynomials

$$
B_{n}^{\alpha, M_{0}, M_{1}}(x)=B_{n}^{\alpha}(x)+\sum_{k=0}^{n-1} a_{n, k} B_{k}^{\alpha}(x), \quad n \geq 1 .
$$

Then we can find the coefficients $a_{n, k}$ using the orthogonality of the polynomials $B_{n}^{\alpha, M_{0}, M_{1}}(x)$ with respect to $\mathcal{L}_{1}$, i.e.,

$$
\begin{aligned}
<\mathcal{L}_{0}, B_{n}^{\alpha, M_{0}, M_{1}}(x) B_{j}^{\alpha}(x)> & =<\mathcal{L}_{0}, B_{n}^{\alpha}(x) B_{j}^{\alpha}(x)>+\sum_{k=0}^{n-1} a_{n, k}<\mathcal{L}_{0}, B_{j}^{\alpha}(x) B_{k}^{\alpha}(x)> \\
& =a_{n, j}<\mathcal{L}_{0},\left(B_{j}^{\alpha}(x)\right)^{2}>, \quad 0 \leq j \leq n-1,
\end{aligned}
$$

whence

$$
a_{n, j}=\frac{<\mathcal{L}_{0}, B_{n}^{\alpha, M_{0}, M_{1}}(x) B_{j}^{\alpha}(x)>}{<\mathcal{L}_{0},\left(B_{j}^{\alpha}(x)\right)^{2}>}, \quad 0 \leq j \leq n-1 .
$$

Since

$$
\begin{aligned}
<\mathcal{L}_{1}, B_{n}^{\alpha, M_{0}, M_{1}}(x) B_{j}^{\alpha}(x)>=0, \quad 0 \leq j<n, & =<\mathcal{L}_{1}, B_{n}^{\alpha, M_{0}, M_{1}}(x) B_{j}^{\alpha}(x)>-M_{0} B_{n}^{\alpha, M_{0}, M_{1}}(0) B_{j}^{\alpha}(0)- \\
& -M_{1}\left(\left(B_{n}^{\alpha, M_{0}, M_{1}}\right)^{\prime}(0) B_{j}^{\alpha}(0)+B_{n}^{\alpha, M_{0}, M_{1}, M_{1}}(0)\left(B_{j}^{\alpha}\right)^{\prime}(0)\right)= \\
& =-M_{0} B_{n}^{\alpha, M_{0}, M_{1}}(0) B_{j}^{\alpha}(0)+M_{1}\left(B_{n}^{\alpha, M_{0}, M_{1}}\right)^{\prime}(0) B_{j}^{\alpha}(0)+ \\
& +M_{1} B_{n}^{\alpha, M_{0}, M_{1}}(0)\left(B_{j}^{\alpha}\right)^{\prime}(0) .
\end{aligned}
$$

If we use the decomposition (25) and substitute the coefficients $a_{n, j}$ according to (27) we obtain an explicit expression for the generalized Bessel polynomial $B_{n}^{\alpha, M_{0}, M_{1}}(x)$

$$
\begin{gathered}
B_{n}^{\alpha, M_{0}, M_{1}}(x)=B_{n}^{\alpha}(x)+\sum_{k=0}^{n-1} \frac{B_{k}^{\alpha}(x)}{<\mathcal{L}_{0},\left(B_{k}^{\alpha}(x)\right)^{2}>} \times \\
\times\left(-M_{0} B_{n}^{\alpha, M_{0}, M_{1}}(0) B_{k}^{\alpha}(0)+M_{1}\left(B_{n}^{\alpha, M_{0}, M_{1}}\right)^{\prime}(0) B_{k}^{\alpha}(0)+M_{1} B_{n}^{\alpha, M_{0}, M_{1}}(0)\left(B_{k}^{\alpha}\right)^{\prime}(0)\right)= \\
=B_{n}^{\alpha}(x)-M_{0} B_{n}^{\alpha, M_{0}, M_{1}}(0) K_{n-1}^{\alpha}(x, 0)+M_{1}\left(B_{n}^{\alpha, M_{0}, M_{1}}\right)^{\prime}(0) K_{n-1}^{\alpha}(x, 0)+ \\
+M_{1} B_{n}^{\alpha, M_{0}, M_{1}}(0) K_{n-1}^{\alpha(0,1)}(x, 0) .
\end{gathered}
$$


Thus (23) follows. In particular,

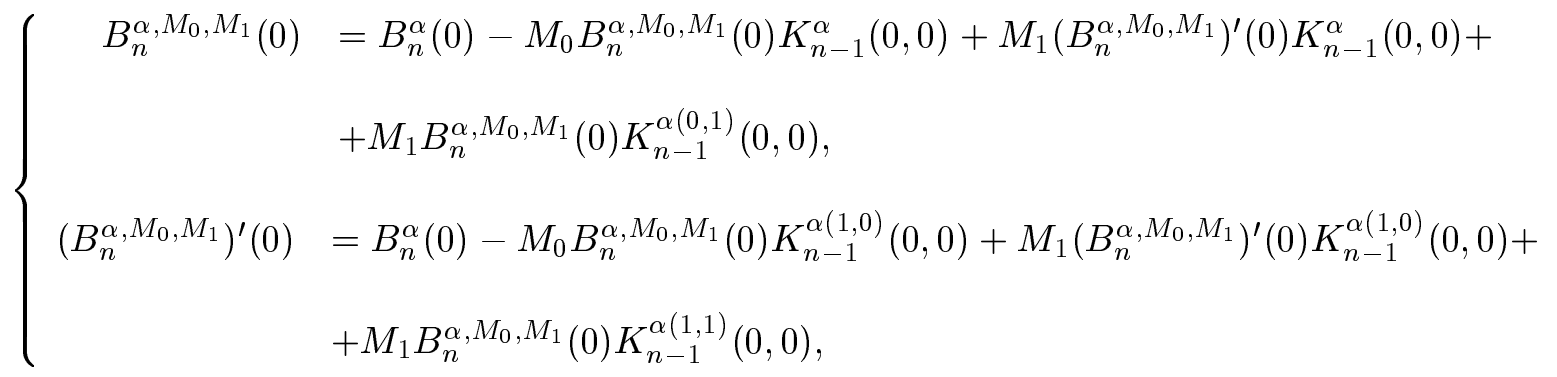

or, equivalently,

$$
\mathbb{K}_{n-1} \cdot\left(\begin{array}{c}
B_{n}^{\alpha, M_{0}, M_{1}}(0) \\
\left(B_{n}^{\alpha, M_{0}, M_{1}}\right)^{\prime}(0)
\end{array}\right)=\left(\begin{array}{c}
B_{n}^{\alpha}(0) \\
\left(B_{n}^{\alpha}\right)^{\prime}(0)
\end{array}\right)
$$

Now,

$$
\begin{aligned}
\mathbb{K}_{n} & =\mathbb{K}_{n-1}+\left(\begin{array}{cc}
M_{0} \frac{\left(B_{n}^{\alpha}(0)\right)^{2}}{<\mathcal{L}_{0},\left(B_{n}^{\alpha}(x)\right)^{2}>}-M_{1} \frac{\left(B_{n}^{\alpha}\right)^{\prime}(0) B_{n}^{\alpha}(0)}{<\mathcal{L}_{0},\left(B_{n}^{\alpha}(x)\right)^{2}>} & -M_{1} \frac{\left(B_{n}^{\alpha}(0)\right)^{2}}{<\mathcal{L}_{0},\left(B_{n}^{\alpha}(x)\right)^{2}>} \\
M_{0} \frac{\left(B_{n}^{\alpha}\right)^{\prime}(0) B_{n}^{\alpha}(0)}{<\mathcal{L}_{0},\left(B_{n}^{\alpha}(x)\right)^{2}>}-M_{1} \frac{\left(B_{n}^{\alpha}\right)^{\prime}(0)\left(B_{n}^{\alpha}\right)^{\prime}(0)}{<\mathcal{L}_{0},\left(B_{n}^{\alpha}(x)\right)^{2}>} & -M_{1} \frac{\left(B_{n}^{\alpha}\right)^{\prime}(0) B_{n}^{\alpha}(0)}{<\mathcal{L}_{0},\left(B_{n}^{\alpha}(x)\right)^{2}>}
\end{array}\right)= \\
& =\mathbb{K}_{n-1}+\frac{1}{<\mathcal{L}_{0},\left(B_{n}^{\alpha}(x)\right)^{2}>}\left(\begin{array}{c}
B_{n}^{\alpha}(0) \\
\left(B_{n}^{\alpha}\right)^{\prime}(0)
\end{array}\right) \cdot\left(\begin{array}{cc}
M_{0} B_{n}^{\alpha}(0)-M_{1}\left(B_{n}^{\alpha}\right)^{\prime}(0), & \left.-M_{1} B_{n}^{\alpha}(0)\right)
\end{array}\right) .
\end{aligned}
$$

Substituting (31) in (32) and computing the determinant on both sides of the equality we get

$$
\frac{\triangle_{n}^{\alpha, M_{0}, M_{1}}}{\triangle_{n-1}^{\alpha, M_{0}, M_{1}}}=1+\frac{B_{n}^{\alpha, M_{0}, M_{1}}(0)\left[M_{0} B_{n}^{\alpha}(0)-M_{1}\left(B_{n}^{\alpha}\right)^{\prime}(0)\right]-\left(B_{n}^{\alpha, M_{0}, M_{1}}\right)^{\prime}(0) M_{1} B_{n}^{\alpha}(0)}{<\mathcal{L}_{0},\left(B_{n}^{\alpha}(x)\right)^{2}>} .
$$

On the other hand,

$$
\begin{aligned}
<\mathcal{L}_{1},\left(B_{n}^{\alpha, M_{0}, M_{1}}(x)\right)^{2}> & =\mathcal{L}_{1}\left[B_{n}^{\alpha, M_{0}, M_{1}}(x) B_{n}^{\alpha}(x)\right]= \\
& =<\mathcal{L}_{0}, B_{n}^{\alpha, M_{0}, M_{1}}(x) B_{n}^{\alpha}(x)>+M_{0} B_{n}^{\alpha}(0) B_{n}^{\alpha, M_{0}, M_{1}}(0)- \\
& -M_{1} B_{n}^{\alpha}(0)\left(B_{n}^{\alpha, M_{0}, M_{1}}\right)^{\prime}(0)-M_{1}\left(B_{n}^{\alpha}\right)^{\prime}(0) B_{n}^{\alpha, M_{0}, M_{1}}(0)= \\
& =<\mathcal{L}_{0},\left(B_{n}^{\alpha}(x)\right)^{2}>+M_{0} B_{n}^{\alpha}(0) B_{n}^{\alpha, M_{0}, M_{1}}(0)- \\
& -M_{1}\left(B_{n}^{\alpha}\right)^{\prime}(0) B_{n}^{\alpha, M_{0}, M_{1}}(0)-M_{1} B_{n}^{\alpha}(0)\left(B_{n}^{\alpha, M_{0}, M_{1}}\right)^{\prime}(0) .
\end{aligned}
$$

From (33)-(34), we deduce

$$
\triangle_{n}^{\alpha, M_{0}, M_{1}}<\mathcal{L}_{0},\left(B_{n}^{\alpha}(x)\right)^{2}>=\triangle_{n-1}^{\alpha, M_{0}, M_{1}}<\mathcal{L}_{1},\left(B_{n}^{\alpha, M_{0}, M_{1}}(x)\right)^{2}>, \quad n \geq 1 .
$$

When $n=0$, since $<\mathcal{L}_{1}, 1>=<\mathcal{L}_{0}, 1>+M_{0} \neq 0$, and

$$
\operatorname{det} \mathbb{K}_{0}=\operatorname{det}\left(\begin{array}{cc}
1+\frac{M_{0}}{<\mathcal{L}_{0}, 1>} & -\frac{M_{1}}{<\mathcal{L}_{0}, 1>} \\
0 & 1
\end{array}\right)=1+\frac{M_{0}}{<\mathcal{L}_{0}, 1>}=\frac{<\mathcal{L}_{1}, 1>}{<\mathcal{L}_{0}, 1>} \neq 0 .
$$

If we get det $\mathbb{K}_{-1}=1$, then (35) holds also for $n=0$. Thus, from (35) it follows by induction that $\operatorname{det} \mathbb{K}_{n} \neq 0, n \geq 0$ and

$$
\triangle_{n}^{\alpha, M_{0}, M_{1}}=\triangle_{n-1}^{\alpha, M_{0}, M_{1}} \frac{<\mathcal{L}_{1},\left(B_{n}^{\alpha, M_{0}, M_{1}}(x)\right)^{2}>}{<\mathcal{L}_{0},\left(B_{n}^{\alpha}(x)\right)^{2}>}, \quad n \geq 0 .
$$


Conversely, assume that $\operatorname{det} \mathbb{K}_{n} \neq 0, n \geq 0$ and define $B_{n}^{\alpha, M_{0}, M_{1}}(x)$ by (23) and (24). Then $\left\{B_{n}^{\alpha, M_{0}, M_{1}}(x)\right\}_{0}^{\infty}$ is a MOPS. Indeed,

$$
\begin{aligned}
<\mathcal{L}_{1}, B_{n}^{\alpha, M_{0}, M_{1}}(x) B_{j}^{\alpha}(x)> & =<\mathcal{L}_{0}, B_{n}^{\alpha, M_{0}, M_{1}}(x) B_{j}^{\alpha}(x)>+M_{0} B_{n}^{\alpha, M_{0}, M_{1}}(0) B_{j}^{\alpha}(0)- \\
& -M_{1}\left(\left(B_{n}^{\alpha, M_{0}, M_{1}}\right)^{\prime}(0) B_{j}^{\alpha}(0)+B_{n}^{\alpha, M_{0}, M_{1}}(0)\left(B_{j}^{\alpha}\right)^{\prime}(0)\right) \\
& =<\mathcal{L}_{0}, B_{n}^{\alpha}(x) B_{j}^{\alpha}(x)>-M_{0} B_{n}^{\alpha, M_{0}, M_{1}}(0)<\mathcal{L}_{0}, K_{n-1}^{\alpha}(x, 0) B_{j}^{\alpha}(x)> \\
& +M_{1}\left(B_{n}^{\alpha, M_{0}, M_{1}}\right)^{\prime}(0)<\mathcal{L}_{0}, K_{n-1}^{\alpha}(x, 0) B_{j}^{\alpha}(x)> \\
& +M_{1} B_{n}^{\alpha, M_{0}, M_{1}}(0)<\mathcal{L}_{0}, K_{n-1}^{\alpha(0,1)}(x, 0) B_{j}^{\alpha}(x)>+M_{0} B_{n}^{\alpha, M_{0}, M_{1}}(0) B_{n}^{\alpha}(0) \\
& -M_{1}\left(\left(B_{n}^{\alpha, M_{0}, M_{1}}\right)^{\prime}(0) B_{j}^{\alpha}(0)+B_{n}^{\alpha, M_{0}, M_{1}}(0)\left(B_{j}^{\alpha}\right)^{\prime}(0)\right) .
\end{aligned}
$$

Hence

$$
\begin{aligned}
<\mathcal{L}_{1}, B_{n}^{\alpha, M_{0}, M_{1}}(x) B_{j}^{\alpha}(x)>=\left\{\begin{array}{ccc}
0 & \text { if } & 0 \leq j \leq n-1, \\
<\mathcal{L}_{0},\left(B_{n}^{\alpha}(x)\right)^{2}>+ & \text { if } & j=n, \\
+M_{0} B_{n}^{\alpha, M_{0}, M_{1}}(0) B_{j}^{\alpha}(0)- & \\
-M_{1}\left(B_{n}^{\alpha, M_{0}, M_{1}}\right)^{\prime}(0) B_{j}^{\alpha}(0)- & \text { if } & 0 \leq j \leq n-1, \\
-M_{1} B_{n}^{\alpha, M_{0}, M_{1}}(0)\left(B_{j}^{\alpha}\right)^{\prime}(0) & 0 & j=n .
\end{array}\right. \\
=\left\{\begin{array}{ccc}
\triangle_{n}^{\alpha, M_{0}, M_{1}}<\mathcal{L}_{0},\left(B_{n}^{\alpha}(x)\right)^{2}>\neq 0 & \text { if }
\end{array}\right.
\end{aligned}
$$

By $(33)$

$$
<\mathcal{L}_{1},\left(B_{n}^{\alpha, M_{0}, M_{1}}(x) B_{j}^{\alpha, M_{0}, M_{1}}(x)>=\left\{\begin{array}{ccc}
0 & \text { if } & 0 \leq j \leq n-1, \\
\neq 0 & \text { if } & j=n .
\end{array}\right.\right.
$$

Thus $\left\{B_{n}^{\alpha, M_{0}, M_{1}}(x)\right\}_{0}^{\infty}$ is a MOPS with respect to $\mathcal{L}_{1}$ and so $\mathcal{L}_{1}$ is quasi-definite.

\section{Representation as hypergeometric series.}

The explicit expression of the Bessel kernels (19)-(20) allows us to write $B_{n}^{\alpha, M_{0}, M_{1}}(x)$ in terms of the classical Bessel polynomials and their first derivatives. In fact, substituting (19)-(20) into (23) we have

$$
B_{n}^{\alpha, M_{0}, M_{1}}(x)=\left[1-n \zeta_{n}^{\alpha, M_{0}, M_{1}}\right] B_{n}^{\alpha}(x)+\left[\eta_{n}^{\alpha, M_{0}, M_{1}}+x \zeta_{n}^{\alpha, M_{0}, M_{1}}\right]\left(B_{n}^{\alpha}(x)\right)^{\prime},
$$


where

$$
\begin{aligned}
\zeta_{n}^{\alpha, M_{0}, M_{1}} & =\frac{(2 n+\alpha)^{2}(2 n+\alpha-1) B_{n}^{\alpha}(0)}{4(n+\alpha) d_{n-1}^{2}} \times \\
& \times\left[M_{0} B_{n}^{\alpha, M_{0}, M_{1}}(0)-M_{1}\left(B_{n}^{\alpha, M_{0}, M_{1}}\right)^{\prime}(0)-\frac{1}{2} n(n+\alpha+1) M_{1} B_{n}^{\alpha, M_{0}, M_{1}}(0)\right], \\
\eta_{n}^{\alpha, M_{0}, M_{1}} & =-\frac{(2 n+\alpha)^{2}(2 n+\alpha-1) B_{n}^{\alpha}(0)}{4(n+\alpha) d_{n-1}^{2}} M_{1} B_{n}^{\alpha, M_{0}, M_{1}}(0) .
\end{aligned}
$$

Taking into account (8) the equation (41) becomes

$$
B_{n}^{\alpha, M_{0}, M_{1}}(x)=\left[1-n \zeta_{n}^{\alpha, M_{0}, M_{1}}\right] B_{n}^{\alpha}(x)+\left[\eta_{n}^{\alpha, M_{0}, M_{1}}+x \zeta_{n}^{\alpha, M_{0}, M_{1}}\right] n B_{n-1}^{\alpha+2}(x) .
$$

On the other hand (see [9, Table VI, page 302]),

$$
B_{n}^{\alpha}(x)=B_{n}^{\alpha+2}(x)+\frac{4 n B_{n-1}^{\alpha+2}(x)}{(2 n+\alpha)(2 n+\alpha+2)}+\frac{4(n-1) n B_{n-2}^{\alpha+2}(x)}{(2 n+\alpha-1)(2 n+\alpha)^{2}(2 n+\alpha+1)},
$$

and

$$
x B_{n-1}^{\alpha+2}(x)=B_{n}^{\alpha+2}(x)-\frac{2(\alpha+2) B_{n-1}^{\alpha+2}(x)}{(2 n+\alpha)(2 n+\alpha+2)}-\frac{4(n-1)(n+\alpha+1) B_{n-2}^{\alpha+2}(x)}{(2 n+\alpha-1)(2 n+\alpha)^{2}(2 n+\alpha+1)} .
$$

Thus, we deduce

$$
B_{n}^{\alpha, M_{0}, M_{1}}(x)=B_{n}^{\alpha+2}(x)+a_{n}^{\alpha, M_{0}, M_{1}} B_{n-1}^{\alpha+2}(x)+b_{n}^{\alpha, M_{0}, M_{1}} B_{n-2}^{\alpha+2}(x),
$$

where

$$
\begin{aligned}
& a_{n}^{\alpha, M_{0}, M_{1}}=\frac{4 n}{(2 n+\alpha)(2 n+\alpha+2)}\left[1+\frac{\eta_{n}^{\alpha, M_{0}, M_{1}}(2 n+\alpha)(2 n+\alpha+2)}{4}-\frac{(2 n+\alpha+2) \zeta_{n}^{\alpha, M_{0}, M_{1}}}{2}\right], \\
& b_{n}^{\alpha, M_{0}, M_{1}}=\frac{4(n-1) n}{(2 n+\alpha-1)(2 n+\alpha)^{2}(2 n+\alpha+1)}\left[1-(2 n+\alpha+1) \zeta_{n}^{\alpha, M_{0}, M_{1}}\right] .
\end{aligned}
$$

Now, we will prove the following proposition.

Proposition 2 The orthogonal polynomial $B_{n}^{\alpha, M_{0}, M_{1}}(x)$ is, up to a constant factor, a generalized hypergeometric series. More precisely,

$$
B_{n}^{\alpha, M_{0}, M_{1}}(x)=\frac{2^{n-2} \pi_{2}(0)}{(n+\alpha+1)_{n+2} n(n-1)}{ }_{4} F_{2}\left(\begin{array}{c}
-n, n+\alpha+1, \beta_{0}+1, \beta_{1}+1 \\
\beta_{0}, \quad \beta_{1}
\end{array} \mid-\frac{x}{2}\right),
$$

where $\pi_{2}(0)$ is given in (53) and the coefficients $-\beta_{0}$ and $-\beta_{1}$ are the solutions of the quadratic equation in $k$ (see formula (50) below) and they are, in general, complex numbers. In the case when for some $i=0,1,-\beta_{i}$ is a negative integer number we need to take the analytic continuation of the hypergeometric series (48).

(48) can be considered as a generalization of the representation of the Bessel polynomials as hypergeometric series.

Proof: Substituting the hypergeometric representation of the Bessel polynomials (17) into (46) we 
find

$$
\begin{aligned}
B_{n}^{\alpha, M_{0}, M_{1}}(x) & =\frac{2^{n-2}}{(n+\alpha+1)_{n+2} n(n-1)} \sum_{k=0}^{\infty}[4 n(n-1)(k+n+\alpha+1)(k+n+\alpha+2)- \\
& -2 a_{n}^{\alpha, M_{0}, M_{1}}(k+n+\alpha+1)(k-n)(n-1)(2 n+\alpha+1)(2 n+\alpha+2)+ \\
& \left.+b_{n}^{\alpha, M_{0}, M_{1}}(k-n+1)(k-n)(2 n+\alpha-1)(2 n+\alpha)(2 n+\alpha+1)(2 n+\alpha+2)\right] \times \\
& \times \frac{(-n)_{k}(n+\alpha+1)_{k}}{k !}\left(-\frac{x}{2}\right)^{k} .
\end{aligned}
$$

Taking into account that the expression inside the quadratic brackets is a polynomial in $k$ of degree 2 and denoting it $\pi_{2}(k)$, we can write

$$
B_{n}^{\alpha, M_{0}, M_{1}}(x)=\frac{2^{n-2} a_{n}}{(n+\alpha+1)_{n+2} n(n-1)} \sum_{k=0}^{\infty} \frac{(-n)_{k}(n+\alpha+1)_{k}\left(k+\beta_{0}\right)\left(k+\beta_{1}\right)}{k !}\left(-\frac{x}{2}\right)^{k},
$$

where $a_{n}$ is the leading coefficient of $\pi_{2}(k)$ :

$$
\begin{aligned}
a_{n} & =4 n(n-1)-2 a_{n}^{\alpha, M_{0}, M_{1}}(n-1)(2 n+\alpha+1)(2 n+\alpha+2)+ \\
& +b_{n}^{\alpha, M_{0}, M_{1}}(2 n+\alpha-1)(2 n+\alpha)(2 n+\alpha+1)(2 n+\alpha+2),
\end{aligned}
$$

and $\beta_{i}=\beta_{i}\left(n, \alpha, \beta, A_{1}, A_{2}, B_{1}, B_{2}\right)(i=0,1)$ are the solutions of the quadratic equation in $k$ (see formula (49)). Since $\left(k+\beta_{i}\right)=\frac{\beta_{i}\left(\beta_{i}+1\right)_{k}}{\left(\beta_{i}\right)_{k}}, i=0,1$ (50) becomes

$$
B_{n}^{\alpha, M_{0}, M_{1}}(x)=\frac{2^{n-2} \pi_{2}(0)}{(n+\alpha+1)_{n+2} n(n-1)} \sum_{k=0}^{\infty} \frac{(-n)_{k}(n+\alpha+1)_{k}\left(1+\beta_{0}\right)_{k}\left(1+\beta_{1}\right)_{k}}{k !\left(\beta_{0}\right)_{k}\left(\beta_{1}\right)_{k}}\left(-\frac{x}{2}\right)^{k}
$$

where

$$
\begin{aligned}
\pi_{2}(0) & =4 n(n-1)(n+\alpha+1)(n+\alpha+2)+ \\
& +2 a_{n}^{\alpha, M_{0}, M_{1}} n(n-1)(n+\alpha+1)(2 n+\alpha+1)(2 n+\alpha+2)+ \\
& +b_{n}^{\alpha, M_{0}, M_{1}} n(n-1)(2 n+\alpha-1)(2 n+\alpha)(2 n+\alpha+1)(2 n+\alpha+2),
\end{aligned}
$$

which, by using the definition (18), is nothing else but the hypergeometric representation (48).

\section{$5 \quad$ Some Asymptotic Formulas.}

In this section we will study some asymptotic formulas for the generalized Bessel polynomials. More precisely, the ratio $B_{n}^{\alpha, M_{0}, M_{1}}(x) / B_{n}^{\alpha}(x)$, outside of the closed contour $\Gamma$ containing the origin and the difference between the new polynomials and the classical ones, inside $\Gamma$. To obtain the first one we will rewrite (41) in the form

$$
\frac{B_{n}^{\alpha, M_{0}, M_{1}}(x)}{B_{n}^{\alpha}(x)}=1-n \zeta_{n}^{\alpha, M_{0}, M_{1}}+\left[\eta_{n}^{\alpha, M_{0}, M_{1}}+x \zeta_{n}^{\alpha, M_{0}, M_{1}}\right] \frac{\left(B_{n}^{\alpha}(x)\right)^{\prime}}{B_{n}^{\alpha}(x)} .
$$


In order to obtain them we will rewrite the kernels (20) as follows:

$$
\begin{aligned}
K_{n-1}^{\alpha}(0,0) & =\frac{(2 n+\alpha)^{2}(2 n+\alpha-1)\left(B_{n}^{\alpha}(0)\right)^{2}}{4(n+\alpha) d_{n-1}^{2}} ; \\
K_{n-1}^{\alpha(0,1)}(0,0) & =\frac{1}{2}(n-1)(n+\alpha+1) K_{n-1}^{\alpha}(0,0) ; \\
K_{n-1}^{\alpha(1,1)}(0,0) & =\frac{1}{4}(n+\alpha+1)[n(n-2)(n+\alpha+1)+\alpha+2] K_{n-1}^{\alpha}(0,0) ;
\end{aligned}
$$

and also we will use the expression $\left(B_{n}^{\alpha}\right)^{\prime}(0)=\frac{1}{2} n(n+\alpha+1) B_{n}^{\alpha}(0)$.

Now, using the asymptotic formula for the Gamma function (see [13, formula 8.16, page 88], [14])

$$
\Gamma(a x+b) \sim \sqrt{2 \pi} e^{-a x}(a x)^{a x+b-\frac{1}{2}}, \quad x>>1, \quad a, b, x \in \mathbb{R},
$$

we find

$$
K_{n-1}^{\alpha}(0,0) \sim \frac{1}{\pi 2^{2 \alpha+1} n^{\alpha-1}}\left(\frac{e}{2 n}\right)^{4 n}, \quad \frac{\left[B_{n}^{\alpha}(0)\right]^{2}}{d_{n-1}^{2}} \sim \frac{(-1)^{n}}{2^{3 \alpha-1} n^{\alpha-2}}\left(\frac{e}{2 n}\right)^{4 n} .
$$

Using the explicit values for $B_{n}^{\alpha, M_{0}, M_{1}}(0)$ and $\left(B_{n}^{\alpha, M_{0}, M_{1}}\right)^{\prime}(0)$ we find

$$
\begin{aligned}
& {\left[M_{0} B_{n}^{\alpha, M_{0}, M_{1}}(0)-M_{1}\left(B_{n}^{\alpha, M_{0}, M_{1}}\right)^{\prime}(0)-\frac{1}{2} n(n+\alpha+1) M_{1} B_{n}^{\alpha, M_{0}, M_{1}}(0)\right]=} \\
& =\frac{\left(4 M_{0}-M_{1}(1+\alpha+n)\left[(1+\alpha) K_{n-1}^{\alpha}(0,0) M_{1}+4 n\right]\right) B_{n}^{\alpha}(0)}{4+K_{n-1}^{\alpha}(0,0)^{2} M_{1}^{2} n(1+\alpha+n)+4 K_{n-1}^{\alpha}(0,0)\left(M_{0}-M_{1}(n-1)(1+\alpha+n)\right)} .
\end{aligned}
$$

From the above expression and (42) we obtain

$$
\begin{aligned}
& \zeta_{n}^{\alpha, M_{0}, M_{1}} \sim \widetilde{C}\left(n, \alpha, M_{0}, M_{1}\right) \frac{(-1)^{n}}{2^{3 \alpha+3} n^{\alpha-1}}\left(\frac{e}{2 n}\right)^{4 n}\left[1+O\left(n^{-1}\right)\right], \\
& \eta_{n}^{\alpha, M_{0}, M_{1}} \sim M_{1} \frac{(-1)^{n}}{2^{3 \alpha+3} n^{\alpha-1}}\left(\frac{e}{2 n}\right)^{4 n}\left[1+O\left(n^{-1}\right)\right],
\end{aligned}
$$

where

$$
\widetilde{C}\left(n, \alpha, M_{0}, M_{1}\right)=\left[2 n^{3}\left(4 M_{0}+M_{1}\left[2-2 \alpha-9 \alpha^{2}\right]\right)-4 M_{1} n^{4}(1+5 \alpha)-8 M_{1} n^{5}\right] .
$$

Finally, from the asymptotics of the Bessel polynomials [6, Eq. (5) page 124]

$$
B_{n}^{\alpha}(z)=\left(\frac{2 n z}{e}\right)^{n} 2^{\alpha+\frac{1}{2}} e^{1 / z}\left[1-\frac{1+6 \alpha\left(\alpha+1+2 z^{-1}\right)+6 z^{-2}}{24 n}+O\left(n^{-2}\right)\right], \quad z \in \mathbb{C} \backslash\{0\} .
$$

we find outside of the closed contour $\Gamma$ containing the origin (see formula (4))

$$
\frac{\left(B_{n}^{\alpha}\right)^{\prime}(z)}{B_{n}^{\alpha}(z)}=\frac{2}{z}\left[1-\frac{2+(3+2 \alpha) z}{2 n z}\right]+O\left(n^{-2}\right) .
$$

Then, from (54) and using all the above asymptotic formulas we obtain the following estimates for the ratio

$$
\frac{B_{n}^{\alpha, M_{0}, M_{1}}(z)}{B_{n}^{\alpha}(z)}=1+\frac{(-1)^{n} e^{4 n}}{n^{4 n+\alpha-5} 2^{4 n+3 \alpha-4}}\left[\frac{M_{1}\left(2-\alpha z+9 \alpha^{2} z\right)-8 M_{0} z}{z}+10 M_{1} \alpha n-4 M_{1} n^{2}+O\left(n^{-1}\right)\right],
$$

where $z \in \mathbb{C} \backslash\{\Omega\}, \Omega=\operatorname{Int} \Gamma$.

The difference between the new polynomials and the classical ones, when $z$ is inside of the closed contour $\Gamma$ containing the origin, is

$$
B_{n}^{\alpha, M_{0}, M_{1}}(z)-B_{n}^{\alpha}(z)=\widetilde{C}\left(n, \alpha, M_{0}, M_{1}\right) \frac{(-1)^{n+1}}{2^{3 \alpha+3} n^{\alpha-1}}\left(\frac{2 n z}{e}\right)^{n} z^{\alpha+2} e^{1 / z}\left[1+O\left(n^{-1}\right)\right] .
$$




\section{Second order differential equation.}

The generalized Bessel polynomials can be given in terms of the Bessel polynomials $B_{n}^{\alpha}(x)$ and their first derivatives (see formula (41)) by means of the representation formula

$$
B_{n}^{\alpha, M_{0}, M_{1}}(x)=c(x ; n) B_{n}^{\alpha}(x)+d(x ; n) \frac{d}{d x} B_{n}^{\alpha}(x),
$$

where $c(x ; n), d(x ; n)$ are polynomials of bounded degree in $x$ with coefficients depending on $n$ :

$$
c(x ; n)=\left[1-n \zeta_{n}^{\alpha, M_{0}, M_{1}}\right], \quad d(x ; n)=\left[\eta_{n}^{\alpha, M_{0}, M_{1}}+x \zeta_{n}^{\alpha, M_{0}, M_{1}}\right] .
$$

Taking derivatives in the above expression and using (2) and (60) we find

$$
\sigma(x) \frac{d}{d x} B_{n}^{\alpha, M_{0}, M_{1}}(x)=e(x ; n) B_{n}^{\alpha}(x)+f(x ; n) \frac{d}{d x} B_{n}^{\alpha}(x),
$$

where

$$
e(x ; n)=\sigma(x) c^{\prime}(x ; n)-\lambda_{n} d(x ; n), \quad f(x ; n)=\sigma(x)\left[c(x ; n)+d^{\prime}(x ; n)\right]-\tau(x) d(x ; n) .
$$

Analogously, if we take derivatives in (62) and use (2) and (60), we obtain

$$
\sigma^{2}(x) \frac{d^{2}}{d x^{2}} B_{n}^{\alpha, M_{0}, M_{1}}(x)=g(x ; n) B_{n}^{\alpha}(x)+h(x ; n) \frac{d}{d x} B_{n}^{\alpha}(x),
$$

where

$$
\begin{aligned}
& g(x ; n)=\sigma(x) e^{\prime}(x ; n)-\sigma^{\prime}(x) e(x ; n)-\lambda_{n} f(x ; n), \\
& h(x ; n)=\sigma(x)\left[e(x ; n)+f^{\prime}(x ; n)\right]-2 \sigma^{\prime}(x) f(x ; n)-\tau(x) f(x ; n) .
\end{aligned}
$$

The above expressions (60), (62), and (64) yield

$$
\left|\begin{array}{ccc}
B_{n}^{\alpha, M_{0}, M_{1}}(x) & c(x ; n) & d(x ; n) \\
\sigma(x) \frac{d}{d x} B_{n}^{\alpha, M_{0}, M_{1}}(x) & e(x ; n) & f(x ; n) \\
\sigma^{2}(x) \frac{d^{2}}{d x^{2}} B_{n}^{\alpha, M_{0}, M_{1}}(x) & g(x ; n) & h(x ; n)
\end{array}\right|=0 .
$$

Expanding the determinant in (66) by the first column, we find the polynomials $B_{n}^{\alpha, M_{0}, M_{1}}(x)$ satisfy a second order differential equation of the form

$$
\tilde{\sigma}(x ; n) \frac{d^{2}}{d x^{2}} B_{n}^{\alpha, M_{0}, M_{1}}(x)+\tilde{\tau}(x ; n) \frac{d}{d x} B_{n}^{\alpha, M_{0}, M_{1}}(x)+\tilde{\lambda}(x ; n) B_{n}^{\alpha, M_{0}, M_{1}}(x)=0,
$$

where

$$
\begin{aligned}
\tilde{\sigma}(x ; n)= & -x^{2}\left\{\left(\zeta_{n}^{\alpha, M_{0}, M_{1}}-1\right) x^{2}+\left[\zeta_{n}^{\alpha, M_{0}, M_{1}}(2 n+\alpha)+\eta_{n}^{\alpha, M_{0}, M_{1}}\left(2+\alpha+(2 n+\alpha) n \zeta_{n}^{\alpha, M_{0}, M_{1}}\right)\right.\right. \\
& \left.\left.-2 \zeta_{n}^{\alpha, M_{0}, M_{1}}\left(n \zeta_{n}^{\alpha, M_{0}, M_{1}}-1\right)\right] x+\left[n \eta_{n}^{\alpha, M_{0}, M_{1}}(n+\alpha+1)+2\left(1-n \zeta_{n}^{\alpha, M_{0}, M_{1}}\right)\right]\right\}
\end{aligned}
$$




$$
\begin{aligned}
& \tilde{\tau}(x ; n)=-(\alpha+2)\left[\zeta_{n}^{\alpha, M_{0}, M_{1}}-1+(2 n+\alpha) \zeta_{n}^{\alpha, M_{0}, M_{1}}\right] x^{3} \\
& +\left\{2\left[1-\zeta_{n}^{\alpha, M_{0}, M_{1}}\left(4+2 \alpha+2 n-3 n \zeta_{n}^{\alpha, M_{0}, M_{1}}-\alpha n \zeta_{n}^{\alpha, M_{0}, M_{1}}\right)\right]\right. \\
& \left.-\eta_{n}^{\alpha, M_{0}, M_{1}}(\alpha+3)\left(2+\alpha+\alpha n \zeta_{n}^{\alpha, M_{0}, M_{1}}+2 n^{2} \zeta_{n}^{\alpha, M_{0}, M_{1}}\right)\right\} x^{2} \\
& -\left[4 \eta_{n}^{\alpha, M_{0}, M_{1}}\left(3+\alpha-2 n \zeta_{n}^{\alpha, M_{0}, M_{1}}+n^{2} \zeta_{n}^{\alpha, M_{0}, M_{1}}\right)+4 \zeta_{n}^{\alpha, M_{0}, M_{1}}\left(n \zeta_{n}^{\alpha, M_{0}, M_{1}}-1\right)\right. \\
& \left.+\eta_{n}^{\alpha, M_{0}, M_{1}^{2}} n(n+\alpha+1)(\alpha+4)\right] x \\
& -2 \eta_{n}^{\alpha, M_{0}, M_{1}}\left[\eta_{n}^{\alpha, M_{0}, M_{1}} n(n+\alpha+1)-2 n \zeta_{n}^{\alpha, M_{0}, M_{1}}+2\right] \\
& \tilde{\lambda}(x ; n)=n(n+\alpha+1)\left\{\eta_{n}^{\alpha, M_{0}, M_{1}{ }^{2}}(n-1)(n+\alpha+2)+2 \eta_{n}^{\alpha, M_{0}, M_{1}}\left(\zeta_{n}^{\alpha, M_{0}, M_{1}}+1\right)\right. \\
& -2 n \eta_{n}^{\alpha, M_{0}, M_{1}} \zeta_{n}^{\alpha, M_{0}, M_{1}}+\left[2 \zeta_{n}^{\alpha, M_{0}, M_{1}}\left(\zeta_{n}^{\alpha, M_{0}, M_{1}}-n \zeta_{n}^{\alpha, M_{0}, M_{1}}+1\right)\right. \\
& \left.+\eta_{n}^{\alpha, M_{0}, M_{1}}\left(4+\alpha-\alpha \zeta_{n}^{\alpha, M_{0}, M_{1}}-2 n \zeta_{n}^{\alpha, M_{0}, M_{1}}+\alpha n \zeta_{n}^{\alpha, M_{0}, M_{1}}+2 n^{2} \zeta_{n}^{\alpha, M_{0}, M_{1}}\right)\right] x \\
& \left.+\left[\zeta_{n}^{\alpha, M_{0}, M_{1}}(2 n+\alpha+1)-1\right]\right\} .
\end{aligned}
$$

The explicit expression for the coefficients in (67) has been obtained by using the algorithm developed in [4]. A simple calculation shows that for $M_{0}, M_{1}$ equal to zero, we recover from the above expressions the SODE for the classical Bessel polynomials.

\section{Three-term recurrence relation for $B_{n}^{\alpha, M_{0}, M_{1}}(x)$.}

Theorem 1 Assume that $\triangle_{n}^{\alpha, M_{0}, M_{1}} \neq 0, n \geq 0$, i.e., $\mathcal{L}_{1}$ is quasi-definite. Then the MOPS $\left\{B_{n}^{\alpha}(x)\right\}_{0}^{\infty}$ related to $\mathcal{L}_{1}$ satisfies a three-term recurrence relation (TTRR)

$$
B_{n+1}^{\alpha, M_{0}, M_{1}}(x)=\left(x-\beta_{n}^{\alpha, M_{0}, M_{1}}\right) B_{n}^{\alpha, M_{0}, M_{1}}(x)-\gamma_{n}^{\alpha, M_{0}, M_{1}} B_{n-1}^{\alpha, M_{0}, M_{1}}(x), \quad n \geq 1 .
$$

Proof: Since $x B_{n}^{\alpha, M_{0}, M_{1}}(x)$ is a polynomial of degree $n+1$, we have

$$
\begin{aligned}
x B_{n}^{\alpha, M_{0}, M_{1}}(x) & =B_{n+1}^{\alpha, M_{0}, M_{1}}(x)+\beta_{n}^{\alpha, M_{0}, M_{1}} B_{n}^{\alpha, M_{0}, M_{1}}(x)+ \\
& +\gamma_{n}^{\alpha, M_{0}, M_{1}} B_{n-1}^{\alpha, M_{0}, M_{1}}(x)+\sum_{k=0}^{n-2} C_{k}^{n} x^{k},
\end{aligned}
$$

where $C_{k}^{n}, k=1,2, \ldots, n-2$, are real coefficients. Taking the indefinite inner product $(\cdot, \cdot)$ associated with the functional $\mathcal{L}_{1}(\alpha \neq-1,-2,-3, \ldots)$

$$
(p, q)=\int_{\Gamma} p(z) q(z) \rho_{0}^{\alpha}(z) d z+M_{0} p(0) q(0)+\left.M_{1}(p(z) q(z))^{\prime}\right|_{z=0},
$$


multiplying by $x^{m}$ both sides of the above expression, and using the orthogonality of the generalized polynomials $B_{n}^{\alpha, M_{0}, M_{1}}(x)$ we find

$$
0=\left(B_{n}^{\alpha, M_{0}, M_{1}}(x), x^{m+1}\right)=\sum_{k=0}^{n-2} C_{k}^{n}\left(1, x^{m+k}\right), \quad m=0,1, \ldots, n-2 .
$$

Since the determinant of the the above linear system in $C_{k}^{n}$ is different from zero (it is, basically the Gram determinant of order $n-1$ for the indefinite inner product (69), see [14, Section 2.2 pages 25-28]), then we deduce that $C_{k}^{n}=0$ for all $k=0,1, \ldots, n-2$.

Thus,

$$
x B_{n}^{\alpha, M_{0}, M_{1}}(x)=B_{n+1}^{\alpha, M_{0}, M_{1}}+\beta_{n}^{\alpha, M_{0}, M_{1}} B_{n}^{\alpha, M_{0}, M_{1}}(x)+\gamma_{n}^{\alpha, M_{0}, M_{1}} B_{n-1}^{\alpha, M_{0}, M_{1}}(x) .
$$

Let us to obtain the coefficients $\beta_{n}^{\alpha, M_{0}, M_{1}}$ and $\gamma_{n}^{\alpha, M_{0}, M_{1}}$ in (70). Let $\tilde{b}_{n}$ be the coefficient of $x^{n-1}$ in the expansion $B_{n}^{\alpha, M_{0}, M_{1}}=x^{n}+\tilde{b}_{n} x^{n-1}+\ldots$. Then, comparing the coefficients of $x^{n}$ in the two hand sides of (70) we find $\beta_{n}^{\alpha, M_{0}, M_{1}}=\tilde{b}_{n}-\tilde{b}_{n+1}$. To calculate $\gamma_{n}^{\alpha, M_{0}, M_{1}}$ it is sufficient to evaluate (70) in $x=0$ and remark that $B_{n+1}^{\alpha, M_{0}, M_{1}}(0) \neq 0(n>>1)$.

In order to obtain a general expression for the coefficient $\beta_{n}^{\alpha, M_{0}, M_{1}}$ we can use the representation formula (41) for the generalized polynomials, in terms of $B_{n}^{\alpha}(x)$ and $\left(B_{n}^{\alpha}(x)\right)^{\prime}$

$$
B_{n}^{\alpha, M_{0}, M_{1}}(x)=\left[1-n \zeta_{n}^{\alpha, M_{0}, M_{1}}\right] B_{n}^{\alpha}(x)+\left[\eta_{n}^{\alpha, M_{0}, M_{1}}+x \zeta_{n}^{\alpha, M_{0}, M_{1}}\right]\left(B_{n}^{\alpha}(x)\right)^{\prime},
$$

Notice that the constants $\zeta_{n}$ and $\eta_{n}$ depend on $n, \alpha, M_{0}, M_{1}$ (see formula (42)). Doing some algebraic calculations we find that

$$
\tilde{b}_{n}=\left(1-\zeta_{n}\right) b_{n}+\eta_{n}
$$

where $b_{n}$ denotes the coefficient of $x^{n-1}$ for the classical monic Bessel polynomials $B_{n}^{\alpha}(x)$ (see formula $(16)$ ), i.e.,

$$
b_{n}=\frac{2(n-1)}{2 n+\alpha} .
$$

Thus, we obtain the following TTRR coefficients for generalized polynomials

$$
\beta_{n}^{\alpha, M_{0}, M_{1}}=\left(1-\zeta_{n}\right) b_{n}-\left(1-\zeta_{n+1}\right) b_{n+1}+\left(\eta_{n}-\eta_{n+1}\right),
$$

and

$$
\gamma_{n}^{\alpha, M_{0}, M_{1}}=-\frac{B_{n+1}^{\alpha, M_{0}, M_{1}}(0)}{B_{n-1}^{\alpha, M_{0}, M_{1}}(0)}-\beta_{n}^{\alpha, M_{0}, M_{1}} \frac{B_{n}^{\alpha, M_{0}, M_{1}}(0)}{B_{n-1}^{\alpha, M_{0}, M_{1}}(0)} .
$$

\section{Quasi-orthogonality and zeros of $B_{n}^{\alpha, M_{0}, M_{1}}(x)$.}

In this section we will study the quasi-orthogonality of the generalized Bessel polynomials as well as some properties of their zeros.

\subsection{The Quasi-orthogonality.}

Because of $x^{2} \mathcal{L}_{1}=x^{2} \mathcal{L}_{0}$, we get

\section{Proposition 3}

$$
x^{2} B_{n}^{\alpha, M_{0}, M_{1}}(x)=\sum_{j=n-2}^{n+2} c_{n, j} B_{j}^{\alpha}(x), \quad n \geq 2,
$$

where

$$
\begin{aligned}
c_{n, j} & =\frac{<\mathcal{L}_{0}, x^{2} B_{n}^{\alpha, M_{0}, M_{1}}(x) B_{j}^{\alpha}(x)>}{<\mathcal{L}_{0},\left(B_{j}^{\alpha}(x)\right)^{2}>}=\frac{<\mathcal{L}_{1}, x^{2} B_{n}^{\alpha, M_{0}, M_{1}}(x) B_{j}^{\alpha}(x)>}{<\mathcal{L}_{0},\left(B_{j}^{\alpha}(x)\right)^{2}>}, \quad n-2 \leq j \leq n+2, \\
c_{n, n-2} & \neq 0 .
\end{aligned}
$$


Proof: From

$$
x^{2} B_{n}^{\alpha, M_{0}, M_{1}}(x)=\sum_{j=0}^{n+2} c_{n, j} B_{j}^{\alpha}(x),
$$

we deduce

$$
\begin{aligned}
c_{n, k}<\mathcal{L}_{0},\left(B_{k}^{\alpha}(x)\right)^{2}> & =<\mathcal{L}_{0}, x^{2} B_{n}^{\alpha, M_{0}, M_{1}}(x) B_{k}^{\alpha}(x)>=<x^{2} \mathcal{L}_{0}, B_{n}^{\alpha, M_{0}, M_{1}}(x) B_{k}^{\alpha}(x)>= \\
& =<x^{2} \mathcal{L}_{1}, B_{n}^{\alpha, M_{0}, M_{1}}(x) B_{k}^{\alpha}(x)>= \\
& =<\mathcal{L}_{1}, B_{n}^{\alpha, M_{0}, M_{1}}(x) x^{2} B_{k}^{\alpha}(x)>=0, \quad k<n-2 .
\end{aligned}
$$

\subsection{Some properties of the zeros.}

Let us denote $\left(\alpha_{k, n}\right)_{k=1}^{n}$ the zeros of the polynomials $B_{n}^{\alpha, M_{0}, M_{1}}(x)$ for $n$ large enough.

Lemma 1 (Kakeya [6, page 77]) Let $p_{n}(z)=\sum_{j=0}^{n} b_{j} z^{j}$ be a polynomial of degree $n$ with positive coefficients and set $\min _{j} \frac{b_{j}}{b_{j+1}}=a_{1}, \max _{j} \frac{b_{j}}{b_{j+1}}=a_{2}$. Then all zeros $\alpha_{k, n}$ of $p_{n}(z)$ satisfy the inequalities $a_{1} \leq\left|\alpha_{k, n}\right| \leq a_{2}$.

In our case, for $n$ large enough, by using the asymptotic formulas (57) we find

$$
\begin{aligned}
& B_{n}^{\alpha, M_{0}, M_{1}}(x)=x^{n}+\frac{2^{n}}{(\alpha+n+1)_{n}} \sum_{k=0}^{n-1}\left(\begin{array}{c}
n \\
k
\end{array}\right)(n+\alpha+1)_{k} \times \\
& \times\left[1+\frac{8 M_{1}(-1)^{n}}{2^{3(\alpha+1)} n^{\alpha-6}}\left(\frac{e}{2 n}\right)^{4 n}+o\left(\frac{e^{n}}{2^{n} n^{4 n}+\alpha-6}\right)\right]\left(\frac{x}{2}\right)^{k} .
\end{aligned}
$$

or, equivalently, using (44) and (57) one deduces that the product $\left(k+\beta_{0}\right)\left(k+\beta_{1}\right)$ for $n$ large enough is positive.

Then, for $n$ large enough, the ratio satisfies the condition

$$
\frac{b_{j}}{b_{j+1}}=\frac{2(k+1)}{(n-k)(n+\alpha+k+1)}
$$

as well as

$$
\frac{2}{n(n+\alpha+1)}<\frac{b_{j}}{b_{j+1}}<\frac{2 n}{2 n+\alpha+1}, \quad \alpha>-1,
$$

which yields $\left|\alpha_{k, n}\right| \leq 1$.

Then, the following proposition holds.

Proposition 4 If $\mathcal{L}_{1}$ is assumed to be a quasi-definite moment functional all the zeros of $B_{n}^{\alpha, M_{0}, M_{1}}(x)$ for $n$ large enough are located inside the unit circle.

Proof: The statement is a consequence of the previous Lemma and the asymptotic relation (57). In fact, by using the representation (60).

\section{Perturbation matrix and eigenvalue problem.}

From

$$
x^{2} \mathcal{L}_{1}=x^{2} \mathcal{L}_{0}
$$


if $\left\{B_{n}^{\alpha}(x)\right\}_{0}^{\infty},\left\{B_{n}^{\alpha, M_{0}, M_{1}}(x)\right\}_{0}^{\infty}$, and $\left\{B_{n}^{\alpha+2}(x)\right\}_{0}^{\infty}$ denote the orthogonal polynomial sequences with respect to $\mathcal{L}_{0}, \mathcal{L}_{1}$, and $x^{2} \mathcal{L}_{0}$, respectively, we can write $B_{n}^{\alpha, M_{0}, M_{1}}(x)$ in terms of $B_{n}^{\alpha+2}(x)$ as in (44). In matrix form

$$
\left(\begin{array}{c}
B_{0}^{\alpha, M_{0}, M_{1}}(x) \\
B_{1}^{\alpha, M_{0}, M_{1}}(x) \\
B_{2}^{\alpha, M_{0}, M_{1}}(x) \\
\vdots \\
B_{n}^{\alpha, M_{0}, M_{1}}(x)
\end{array}\right)=\left(\begin{array}{ccccccc}
1 & 0 & 0 & \ldots & 0 & 0 & 0 \\
a_{1} & 1 & 0 & \ldots & 0 & 0 & 0 \\
b_{2} & a_{2} & 1 & \ldots & 0 & 0 & 0 \\
\vdots & \vdots & \vdots & \ldots & \vdots & \vdots & \vdots \\
0 & 0 & 0 & \ldots & b_{n} & a_{n} & 1
\end{array}\right) \cdot\left(\begin{array}{c}
B_{0}^{\alpha+2}(x) \\
B_{1}^{\alpha+2}(x) \\
B_{2}^{\alpha+2}(x) \\
\vdots \\
B_{n}^{\alpha+2}(x)
\end{array}\right)
$$

or, equivalently,

$$
\mathbb{Q}_{n}=\mathrm{T}_{n} \cdot \overline{\mathbb{P}}_{n}
$$

where $\mathbb{Q}_{n}$ and $\overline{\mathbb{P}}_{n}$ denote the column vectors of (79), respectively.

On the other hand, from the three-term recurrence relation that $\left\{B_{n}^{\alpha, M_{0}, M_{1}}(x)\right\}_{0}^{\infty}$ and $\left\{B_{n}^{\alpha+2}(x)\right\}_{0}^{\infty}$ satisfy, one gets the matrix representation

$$
\begin{aligned}
x \mathbb{Q}_{n}= & \underbrace{\left(\begin{array}{ccccc}
\tilde{\beta}_{0} & 1 & 0 & \ldots & 0 \\
\tilde{\gamma}_{1} & \tilde{\beta}_{1} & 1 & \ldots & 0 \\
\vdots & \vdots & \vdots & \ldots & \vdots \\
0 & 0 & 0 & \ldots & \tilde{\beta}_{n}
\end{array}\right)}_{\tilde{\mathbf{J}}_{n}} \cdot \mathbb{Q}_{n}+B_{n+1}^{\alpha, M_{0}, M_{1}}(x)\left(\begin{array}{c}
0 \\
\vdots \\
0 \\
1
\end{array}\right), \\
x \overline{\mathbb{P}}_{n} & =\underbrace{\left(\begin{array}{ccccc}
\bar{\beta}_{0} & 1 & 0 & \ldots & 0 \\
\bar{\gamma}_{1} & \bar{\beta}_{1} & 1 & \ldots & 0 \\
\vdots & \vdots & \vdots & \ldots & \vdots \\
0 & 0 & 0 & \ldots & \bar{\beta}_{n}
\end{array}\right)}_{\overline{\mathbf{J}}_{n}} \cdot \overline{\mathbb{P}}_{n}+B_{n+1}^{\alpha+2}(x)\left(\begin{array}{c}
0 \\
\vdots \\
0 \\
1
\end{array}\right) .
\end{aligned}
$$

Substituting (80) into (81) we have

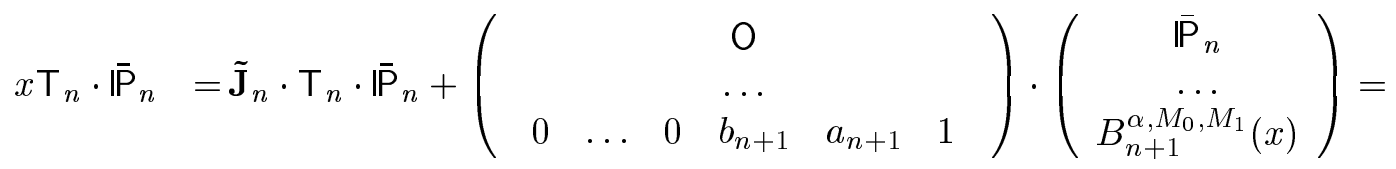

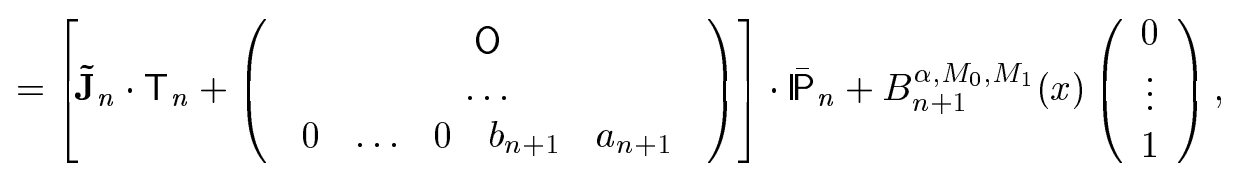

where the matrices $\tilde{\mathbf{J}}_{n}$ and $\overline{\mathbf{J}}_{n}$ are defined in (81)-(82) and $\mathrm{O}$ is the null matrix. Therefore

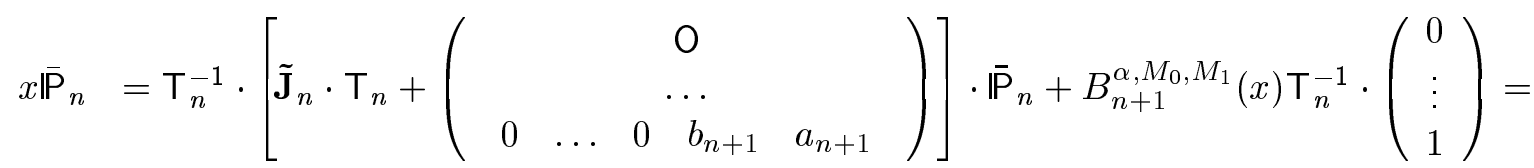

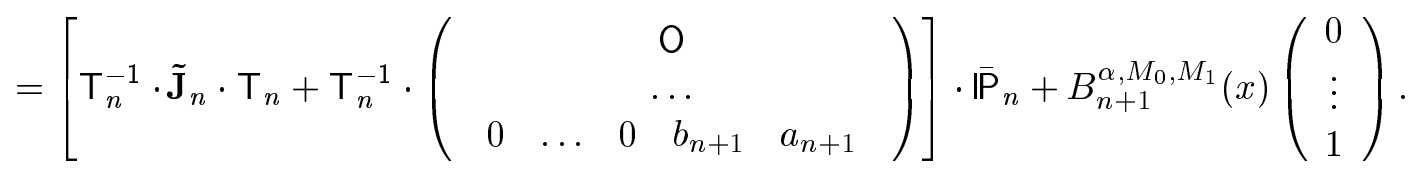


Taking into account (82) we deduce that

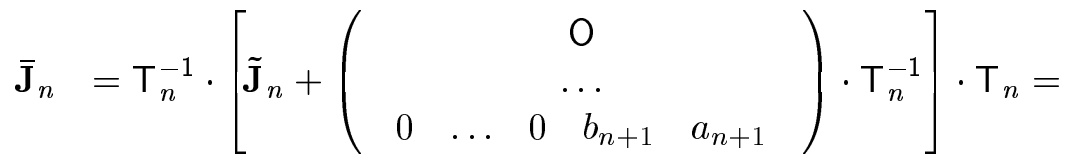

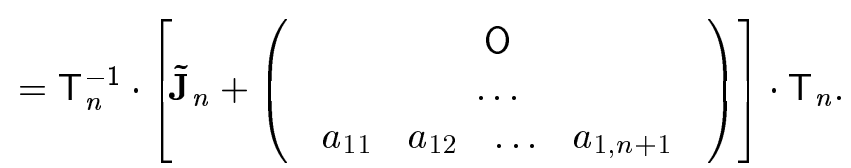

Thus, $\overline{\mathbf{J}}_{n}$ is, essentially, a rank one perturbation of the matrix $\tilde{\mathbf{J}}_{n}$. From (85) notice that

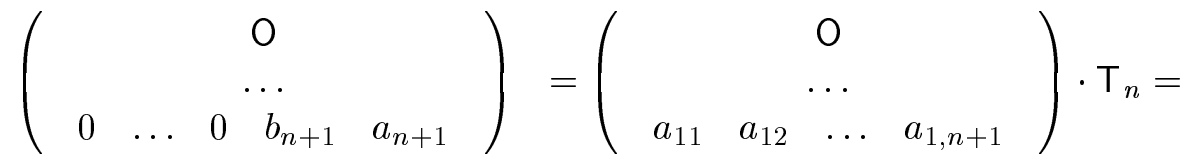

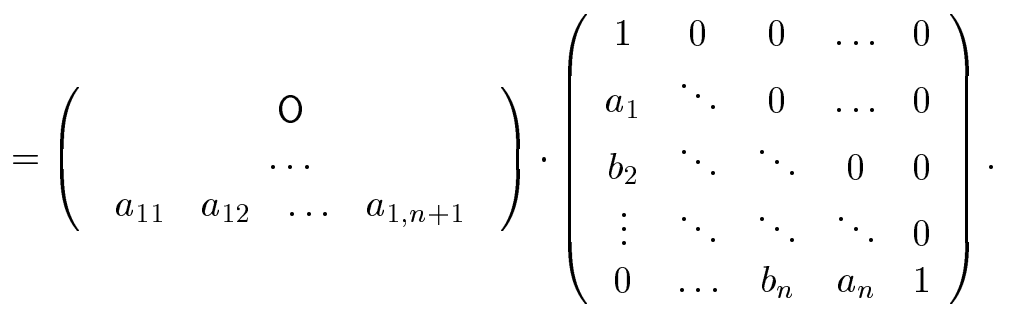

This means

$$
\left\{\begin{array} { c } 
{ a _ { 1 , n + 1 } = a _ { n + 1 } , } \\
{ a _ { 1 , n } + a _ { 1 , n + 1 } a _ { n } = b _ { n + 1 } , } \\
{ a _ { 1 , n - 1 } + a _ { 1 , n } a _ { n - 1 } + a _ { 1 , n + 1 } b _ { n } = 0 , } \\
{ a _ { 1 , n - 2 } + a _ { 1 , n - 1 } a _ { n - 2 } + a _ { 1 , n } b _ { n - 1 } = 0 , } \\
{ \ldots } \\
{ a _ { 1 , 1 } + a _ { 1 , 2 } a _ { 1 } + a _ { 1 , 3 } b _ { 2 } = 0 . }
\end{array} \Leftrightarrow \left\{\begin{array}{c}
a_{1, n+1}=a_{n+1}, \\
a_{1, n}=b_{n+1}-a_{n+1} a_{n}, \\
a_{1, n-1}=-a_{n+1} b_{n}-a_{n-1}\left[b_{n+1}-a_{n+1} a_{n}\right], \\
=-b_{n} a_{n+1}-b_{n+1} a_{n-1}+a_{n-1} a_{n} a_{n+1}, \\
\ldots
\end{array} .\right.\right.
$$

Then, for the rank one matrix, the entries of the last row can be generated in a straightforward way. Let

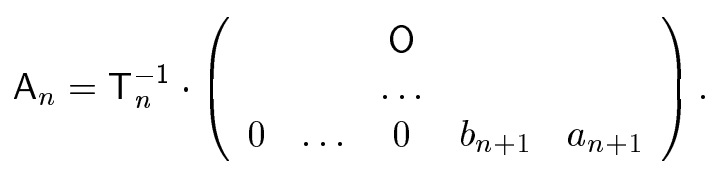

Taking into account that $T_{n}$ is a lower triangular matrix, we deduce that the product matrix has the same structure as the matrix of the second factor on the previous expression, i.e,

$$
\mathrm{A}_{n}=\left(\begin{array}{ccccc} 
& 0 & & \\
& & \ldots & & \\
0 & \ldots & 0 & b_{n+1} & a_{n+1}
\end{array}\right),
$$

Hence

$$
\overline{\mathbf{J}}_{n}=\mathrm{T}_{n}^{-1} \cdot \tilde{\mathbf{J}}_{n} \cdot \mathrm{T}_{n}+\mathrm{A}_{\mathbf{n}}
$$




\section{ACKNOWLEDGEMENTS}

The work of the first three authors was supported by Dirección General de Enzeñanza Superior (DGES) of Spain under grant PB 96-0120-C03-01. The fourth author (KHK) thanks Professor Marcellán for the opportunity to visit University Carlos III de Madrid in January 1997. In addition, he also thanks KOSEF(95-070-02-01-3) and Korea Ministry of Education (BSRI 1420) for their research support.

\section{References}

[1] R. Álvarez-Nodarse and F. Marcellán: A generalization of the classical Laguerre polynomials. Rend. Circ. Mat. Palermo. Serie II, 44, (1995), 315-329.

[2] R. Álvarez-Nodarse and F. Marcellán : A Generalization of the Classical Laguerre Polynomials: Asymptotic Properties and zeros. Appl. Analysis 62, (1996), 349-366.

[3] R. Álvarez-Nodarse, J. Arvesú, and F. Marcellán : A Generalization of the Jacobi-Koornwinder Polynomials. (Submitted)

[4] R. Álvarez-Nodarse and A. Zarzo: On some modifications of classical orthogonal polynomials: differential and spectral properties. (Submitted).

[5] S. Belmehdi and F. Marcellán: Orthogonal polynomials associated to some modifications of a linear functional. Appl. Analysis. 46, (1992), 1-24.

[6] E. Grosswald:Bessel polynomials. Lecture Notes in Math. 698, Springer-Verlag, Berlin, (1978).

[7] E. Hendriksen:A Bessel orthogonal polynomial system. Proc. Kon. Nederl. Acad. Wetensch. 87, (1984) 407-414.

[8] H. L. Krall and O. Frink: A new class of orthogonal polynomials: the Bessel polynomials. Trans. Amer. Math. Soc. 65, (1949), 100-115.

[9] F. Marcellán, A. Branquinho, and J. Petronilho: Classical Orthogonal Polynomials: A Functional Approach. Acta Appl. Math., 34, (1994), 238-303.

[10] F. Marcellán and P. Maroni: Sur l'adjonction d'une masse de Dirac à une forme régulière et semi-classique. Ann. Mat. Pura ed Appl., IV, CLXII, (1992), 1-22.

[11] F. Marcellán and A. Ronveaux : On a class of polynomials orthogonal with respect to a discrete Sobolev inner product. Indag. Math. (N.S.) 1, (1990), 451-464.

[12] F. Marcellán, T.E. Pérez, and M.A. Piñar: Regular Sobolev type orthogonal polynomials: The Bessel case. Rocky Mountain J. of Math. 25, (1995), 1431-1457.

[13] F. W. J. Olver: Asymptotics and Special Functions. Academic Press Inc., New York, 1974.

[14] G. Szegö: Orthogonal Polynomials. Amer. Math. Soc. Colloq. Publ., 23, Amer. Math. Soc., Providence, Rhode Island, 1975 (4th edition).

J. Arvesú ${ }^{\dagger}$ E-mail: arvesu@dulcinea.uc3m.es

R. Álvarez-Nodarse ${ }^{\dagger, *}$ E-mail: renato@dulcinea.uc3m.es

F. Marcellán ${ }^{\dagger}$ E-mail: pacomarc@ing.uc3m.es

K. H. Kwon** E-mail: khkwon@jacobi.kaist.ac.kr

† Departamento de Matemáticas. Escuela Politécnica Superior.

Universidad Carlos III de Madrid. Butarque 15, 28911, Leganés, Madrid.

* Instituto Carlos I de Física Teórica y Computacional

Universidad de Granada E-18071, Granada

** Department of Mathematics, KAIST, Taejon 305-701, Korea 